Gynecologic and

Obstetric Investigation
Received: October 5, 2007

Accepted after revision: January 28, 2008

Published online: April 29, 2008

\title{
Successful Use of Balloon Ablation to Treat Menorrhagia Complicating Aplastic Anemia
}

\author{
Nancy Kim ${ }^{a}$ Theresa Donohue ${ }^{b}$ Elaine Sloand ${ }^{b}$ Pamela Stratton $^{a}$

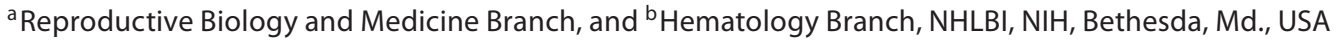

\section{Key Words}

Aplastic anemia $\cdot$ Menorrhagia $\cdot$ Endometrial ablation tology, and that sufficient blood products are available to maintain platelet counts above $30,000 / \mu$ l during the healing process.

\begin{abstract}
Background: Aplastic anemia (AA) complicated by menorrhagia is treated with transfusion and hormonal therapy. When bleeding is life-threatening, balloon endometrial ablation can safely be used to treat menorrhagia in selected patients. Case: A 56-year-old postmenopausal woman was diagnosed with AA after several weeks of menorrhagia and pancytopenia. She became heavily alloimmunized after extensive platelet transfusion. During treatment with antithymocyte globulin, vaginal bleeding increased and the platelet count fell to $1,000 / \mu$ l on supportive measures. After bleeding stopped with use of intravenous Premarin, she was examined in the operating room. There, a clot was removed and appeared to be a uterine caste; hemostasis continued. Transvaginal ultrasound revealed a normal endometrial contour and thin endometrium; endometrial histology was benign. After she completed antithymocyte globulin and her platelet count could be maintained over $30,000 / \mu$ l with matched platelets, endometrial ablation was performed without any complications. Conclusion: Thermal balloon endometrial ablation is an effective alternative to hysterectomy for women with persistent menorrhagia and AA when supportive measures fail. Prior to endometrial ablation, evaluation should ensure normal endometrial contour and his-
\end{abstract}

\section{KARGER}

Fax +41613061234

E-Mail karger@karger.ch

www.karger.com
(C) 2008 S. Karger AG, Basel www.karger.com/goi

\section{Introduction}

Aplastic anemia (AA) is a bone marrow failure disorder characterized by pancytopenia and hypocellular bone marrow. Matched hematopoietic stem cell transplantation and immunosuppression with antithymocyte globulin (ATG) and cyclosporine (CsA) has been extraordinarily successful in producing clinical responses and prolonging survival of patients with AA [1]. Regardless of the type of treatment, patients with severe disease require extensive supportive care with transfusions, hormones, growth factors, and antibiotics to mitigate the infections and bleeding from pancytopenia.

Patients with thrombocytopenia frequently present with bleeding. In particular, menorrhagia is commonly reported in reproductive-aged women with AA and low platelet counts, and may occur, in some, after menopause [2]. This uterine bleeding is managed by the use of transfusions (of both packed red cells and platelets) and hormones which, when used together, often mitigate the bleeding. Also, when treatment for AA is effective, platelet and red cell counts increase, and abnormal bleeding is
Dr. Pamela Stratton

Gynecology Consult Service

Reproductive Biology and Medicine Branch, NICHD/NIH

Bldg 10 CRC, Room 1-3140, Bethesda, MD 20982-1109 (USA)

Tel. +1 301496 9079, Fax +1 301480 6703, E-Mail strattop@mail.nih.gov 
controlled. However, the platelet count may take weeks or months to respond to therapy and the patient often remains red cell and platelet transfusion dependent during this period and may become alloimmunized [3].

Endometrial ablation is a surgical technique used to treat menorrhagia in reproductive-aged women with and without underlying hematologic diseases [4]. New balloon systems for endometrial ablation are relatively safe and easy to use, in those women who have a normal sized cavity without anatomic or histologic abnormalities. While balloon systems were developed to decrease menstrual blood loss in healthy reproductive-aged women, the thermal balloon endometrial ablation has successfully treated menorrhagia in a woman with a bleeding diathesis, who was not a surgical candidate for hysterectomy [5].

We report a case of a postmenopausal woman who was alloimmunized and developed nearly fatal intractable vaginal bleeding from a thin, normal contoured endometrium in the context of AA. She was successfully treated with thermal endometrial ablation.

\section{Case Report}

A 56-year-old postmenopausal woman presented to the Emergency Room after several weeks of heavy vaginal bleeding. An endometrial aspiration under anesthesia revealed a benign endometrial histology. The patient's vaginal bleeding was difficult to control because the patient became heavily alloimmunized after extensive platelet transfusion and eventually did not respond to platelets. As vaginal bleeding continued over the next month, the patient was treated with at least 35 units of red blood cells and received numerous units of platelets. A bone marrow examination revealed severe AA and she was referred to the National Institutes of Health for further evaluation and treatment.

Treatment for AA was initiated with ATG therapy (horse ATG) for 4 days. Several hours after the first dose of ATG, which is known to worsen thrombocytopenia, the patient's vaginal bleeding increased significantly and her platelet count dropped precipitously to $3,000 / \mu \mathrm{l}$. HLA best-matched platelets were procured by the blood bank but were in short supply given the patient's relatively rare HLA type. After consultation with gynecology, megestrol acetate $20 \mathrm{mg}$ daily was started to hormonally suppress the vaginal bleeding.

On the second day of therapy, as vaginal bleeding continued to be heavy, her platelet count dropped to $1,000 / \mu l$ and hematocrit fell to $20 \%$. The vaginal bleeding slowed with $0.2 \mathrm{mg}$ intravenous Premarin (Wyeth Pharmaceuticals Inc., Philadelphia, Pa., USA) and the patient was examined under anesthesia in the operating room. Intraoperative transvaginal ultrasound revealed an endometrial thickness of $1.5 \mathrm{~cm}$. On speculum examination, a clot, seen at the cervical os, was removed using gentle traction. The clot, shaped like the endometrial cavity, appeared to be a uterine caste. The uterine bleeding stopped. Transvaginal ultrasound, af- ter clot removal, revealed the endometrial thickness was $<3 \mathrm{~mm}$, the uterine cavity measured $8 \mathrm{~cm}$ in length and the endometrial contour was smooth on gentle endometrial curettage. Histologic evaluation of the surgical specimen revealed only an organized blood clot.

The vaginal bleeding decreased after the use of intravenous Premarin and removal of the blood clot; however she continued to require several units of blood and HLA-matched platelets to maintain her blood counts. Oral Premarin $(1.25 \mathrm{mg})$ was administered every $8 \mathrm{~h}$ for the first day and then decreased to every $12 \mathrm{~h}$ for 2 weeks. Megestrol acetate was discontinued. Over the next few days, she completed treatment with ATG.

When sufficient HLA-matched donors were recruited and platelets were made available to transfuse to maintain the platelet count over 30,000/ $\mu$ l, endometrial ablation using the Thermachoice $^{\mathrm{TM}}$ (Gynecare, Somerville, N.J., USA) was performed without any complications in a manner similar to the manufacturer's instructions. After the vagina was cleansed with betadine and prophylactic antibiotics were given, the uterine cavity was measured with a uterine sound. The balloon system was inserted through the cervix into the uterine cavity to $1 \mathrm{~cm}$ less than the uterine depth; no tenaculum was needed. Then, the balloon was inflated with sterile $5 \%$ dextrose in water expanding it until the target pressure of 160-180 was obtained. The fluid was then heated to $87^{\circ} \mathrm{C}$ (range $75-90$ ) and circulated for $8 \mathrm{~min}$, maintaining the pressure in the target range. Next the fluid was withdrawn and the catheter removed. After ablation, the platelet count was maintained over 30,000/ $\mu \mathrm{l}$, transfusing additional HLA-matched platelets as needed. Within several days, the bleeding subsided completely.

A week later, she was started on CsA with therapeutic levels. Six weeks following therapy for AA, the patient's blood counts responded and she had an unsupported platelet count of $58,000 / \mu \mathrm{l}$, absolute neutrophil count $1,300 / \mu \mathrm{l}$, and absolute reticulocyte

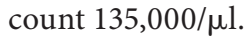

One year later, AA relapsed, but she had no vaginal bleeding. She was treated again with immunosuppressive therapy. Two years after the second therapeutic intervention, she remains on low-dose CsA with completely normalized blood counts and no vaginal bleeding.

\section{Comment}

Menorrhagia in women with AA is rarely life-threatening as transfusion and hormonal treatments are usually effective in stabilizing the patient until counts are restored with medical treatments for AA. Alloimmunization to platelets can occur, especially in patients with many transfusions [4]. In this postmenopausal patient, unrelenting menorrhagia occurred as a result of low platelet counts in the setting of AA; the continued blood loss could not be stopped with the usual supportive measures and hysterectomy was not an option because of her low counts. 
Because the uterus is involuted and atrophic after menopause, it is unusual for postmenopausal women with AA to present with menorrhagia. The pathophysiology of menorrhagia in reproductive-aged women often differs from that of postmenopausal women [6]. The former may have anatomic causes such as fibroids, adenomyosis, or polyps, or endocrinologic causes that cause anovulation and lead to a thickened endometrium. By contrast, postmenopausal women may bleed from endometrial polyps or cancer and are less likely to have hormonal causes unless they are taking exogenous hormones. Thus, patients with AA and difficult to treat menorrhagia should be evaluated for these other causes.

Hormonal therapies have long been recognized as helpful to control menstrual bleeding. While it is not known how intravenous Premarin works in this setting, vasospasm or altering capillary fragility, and then, over a couple of days, reepithelializing the endometrium through the effect of estrogen on endometrial growth are all possibilities [6]. Similarly, combined oral contraceptives and progestins also affect the endometrium by transforming it to a progestin-dominated histology of decidualized stroma and thinning the endometrium [6]. Combined oral contraceptives, often used to prevent or treat menorrhagia in reproductive-aged women, may not be used in older, postmenopausal women [7]. Instead, bleeding might be treated with progestins such as megestrol acetate because of the relative contraindication to estrogens and combined hormonal contraceptive use in postmenopausal women [2]. In this patient, however, hormonal suppression was ineffective and intravenous Premarin was used emergently for a short period.

Thermal endometrial ablation has a short procedure time and is safe and easy to perform even in high-risk surgical patients $[4,5]$. Balloon systems differ mainly in whether the heating element and thermistor is contained within the balloon (Thermachoice, Gynecare, Cincinnati, Ohio) or externally such that glycine or saline is heated before being circulated in the balloon (Cavaterm, Medical Dynamics, The Netherlands, and Menotreat, Lina Medical, Denmark). Other types of global endometrial ablation include cryoablation, impedance bipolar radiofrequency ablation (NovaSure, Cytyc, Marlborough, Mass., USA), microwave endometrial ablation (MEA, Microsulis Medical, UK). These new techniques are associated with a short operative time and greater likelihood for local anesthesia use in selected patients and thus are more readily available to patients who need alternative treatment for menorrhagia [4].
Anesthesia for thermal ablation in this setting may be either local or general. While some anesthesiologists may be reluctant to use general anesthesia in these patients, our anesthesiologists favor general anesthesia in this tertiary care setting as it enables them to rapidly stabilize the patient should complications occur. However, among surgeons experienced with endometrial ablation, local anesthesia is a reasonable alternative.

It is important to delay thermal ablation until sufficient supply of blood products is available and to recognize and be prepared to treat ablation device complications such as perforation. In this case, surgery was delayed until after the ATG therapy was completed and a continued supply of HLA-matched platelets was available before and after the procedure such that blood counts could be stabilized. Complications were minimized by having performed a transvaginal ultrasound to confirm endometrial contour and location.

Patients should be counseled about the options and thermal ablation should be considered in patients like this one. Patients with life-threatening menorrhagia and pancytopenia may need emergent control of excessive vaginal bleeding. While thermal ablation is not specifically recommended for women with intractable bleeding from blood dyscrasias nor does the product label include use in postmenopausal women, in carefully selected patients, ablation may be the best option. For example, among those in whom hysterectomy is not an option because of brisk bleeding, low platelet count and anemia in a setting of a normal sized uterus with a normal contour and known, benign endometrial histology, thermal balloon endometrial ablation offers a reasonable, safe and effective alternative. In this case, endometrial ablation was life-saving.

\section{Acknowledgments}

We would like to thank Sheila Mahoney, CNM, and Dr. James Segars for their assistance in the gynecologic care of this patient. This research was supported by the Intramural Research Program of the NIH, the National Institute of Child Health and Human Development, the National Heart, Lung and Blood Institute and the Clinical Center of the National Institutes of Health. 


\section{References}

Bhatt A, Chenoy R: Menorrhagia due to Bernard-Soulier syndrome and its successful treatment by thermal balloon endometrial ablation. BJOG 2001;108:667-668.

concepts in the pathophysiology and treatment of aplastic anemia. Blood 2006;108: 2509-2519.

6 Jabbour HN, Kelly RW, Fraser HM, CritchGynaecological and obstetric management of women with inherited bleeding disorders. J Obstet Gynaecol Can 2005;27:707-732.

-3 Rebulla P: Refractoriness to platelet transfusion. Curr Opin Hematol 2002;9:516-520.

4 Sharp HT: Assessment of new technology in the treatment of idiopathic menorrhagia and uterine leiomyomata. Obstet Gynecol 2006; 108:990-1003. tion. Endocr Rev 2006;27:17-46.

7 Seibert C, Barbouche E, Fagan J, Myint E, Wetterneck T, Wittemyer M: Prescribing oral contraceptives for women older than 35 years of age. Ann Intern Med 2003;138:5464.

Kim/Donohue/Sloand/Stratton 